\title{
Some Regular Sums*
}

\author{
P. Patrício ${ }^{\dagger}$ and R.E. Hartwig ${ }^{\ddagger}$
}

\begin{abstract}
In this paper, we examine the question of regularity of sums of special elements that appear in the study of orthogonality and invertibility.
\end{abstract}

Keywords: Outer inverses, reflexive inverses, Pierce decomposition, inverse, group inverse, block matrices

AMS classification: 15A09, 16E50

\section{Introduction}

A fundamental problem in matrix theory, and more generally in ring theory, is the creation of units. For special elements such as nilpotents $n$, or idempotents $e$, some associated units are well known, such as $(1-n)^{-1}=1+n+n^{2}+. .+n^{k-1}$ when $n^{k}=0$ or $(1+e x(1-e))^{-1}$, where $e^{2}=e$.

The search for units is facilitated by the existence of some special elements. In particular regular elements can be used, and the corresponding theory of generalized inverses may also be employed, leading to the search for group and Drazin inverses. Here we shall examine the relation between the various types of "invertibility".

Let $R$ be an associative ring with unity 1 throughout the discussions.

The right and left indices of an element $a \in R$, if any, are respectively defined by

$$
\operatorname{rind}(a)=\min \left\{k ; a^{k+1} R=a^{k} R\right\}
$$

and

$$
\operatorname{lind}(a)=\min \left\{k ; R a^{k+1}=R a^{k}\right\} .
$$

If both indices are finite then they are equal, and the element is called strongly-pi-regular. This occurs exactly when there is a unique common solution $x$ to the equations

$$
a^{k} x a=a^{k}, a x=x a, x a x=x,
$$

${ }^{*}$ This research was financed by FEDER Funds through "Programa Operacional Factores de Competitividade - COMPETE" and by Portuguese Funds through FCT - "Fundação para a Ciência e a Tecnologia", within the project PEstC/MAT/UI0013/2011.

${ }^{\dagger}$ CMAT - Centro de Matemtica and Departamento de Matemática e Aplicações, Universidade do Minho, 4710-057 Braga, Portugal. e-mail: pedro@math.uminho.pt

Corresponding author.

${ }^{\ddagger}$ Mathematics Department, N.C.S.U., Raleigh, NC 27695-8205, U.S.A. e-mail: hartwig@unity.ncsu.edu 
for some $k$, and it is denoted by $a^{d}$, the Drazin inverse of $a$.

An element $a$ is regular if $a a^{-} a=a$ for some inner or 1-inverse $a^{-}$. The set of all inner inverses of $a$ is denoted by $\{a\}^{-}$.

We say $\hat{a}$ is an outer inverse of $a$ if $\hat{a} a \hat{a}=\hat{a}$.

An element $a$ has a reflexive inverse $a^{+}$if

$$
a a^{+} a=a, \quad a^{+} a a^{+}=a^{+} .
$$

A reflexive inverse that commutes with $a$, if any, must be unique and is called the group inverse of $a$, denoted by $a^{\#}$.

The method used for studying units and regularity can be multiplicative or additive. The former is limited by the fact that if $a b$ is a unit then $a$ and $b$ have one-sided inverses, but may not be units. On the other hand the shifts $1-a b$ and $1-b a$ are related via

$$
(1-a b)^{-}=1+a(1-b a)^{-} b \quad \text { and } \quad(1-a b)^{-1}=1+a(1-b a)^{-1} b, \text { if any. }
$$

Moreover [2]

$$
(1-a b)^{+}=1+a\left[(1-b a)^{+}-p q\right]^{+} b,
$$

where $p=1-(1-b a)^{+}(1-b a)$ and $q=1-(1-b a)(1-b a)^{+}$.

In the additive case we may employ

(i) special elements;

(ii) (bi)-orthogonality: $a b=0=b a$ and Pierce's decomposition.

(iii) +-orthogonality: $a^{+} b=0=b a^{+}$, which is equivalent to $a R \cap b R=(0)=R a \cap R b$.

Of special interest are units of the form $a+(1-e) h$ where $e$ is idempotent. In particular this appears in the search for group inverse of a product $p a q$, where $a$ is regular and $p, q$ are units. Indeed, [11]:

$$
m=p a q \text { has a group inverse if } u=a+\left(1-a a^{-}\right) p^{-1} q^{-1} \text { is a unit. }
$$

For matrices there are two approaches one can use:

(i) use an "internal" approach in which the resulting elements become "projected", and closer to orthogonal;

(ii) an "external" approach, in which the resulting elements become "more sparse" and hence easier to handle. Again regularity of some of the elements is crucial.

We begin with the "horizontal" question.

\section{Units and bi-orthogonality}

We recall that a large class of additive units is given by the following.

Lemma 2.1. Let $e$ be an idempotent The following are equivalent. 
1. $u=e+(1-e) b$ is a unit.

2. $v=e+(1-e) b(1-e)$ is a unit.

3. $w=e+b(1-e)$ is a unit.

4. $(1-e) b(1-e) R=(1-e) R$ and $R(1-e)=R(1-e) b(1-e)$.

Proof. Since $[1+(1-e) b e] v=u=w[1+e b(1-e)]$, the equivalence of the first three conditions is clear. The fact that $v$ is a unit iff (4) holds, is also well known.

More generally given elements $a$ and $b$, it means that we must find $\alpha, \beta$ such that

$$
(a+\alpha)(b+\beta)=1=(b+\beta)(a+\alpha) .
$$

The simplest case is where we desire/force bi-orthogonality, i.e.

$$
a \beta=0=\beta a \text { and } \alpha b=0=b \alpha .
$$

This leaves

$$
a b+\alpha \beta=1=b a+\beta \alpha .
$$

A possible way of creating orthogonality is by using idempotents $e$ and $f$, not necessarily equal. Our aim is to "transfer" units from the skew-corner ring $(1-e) R(1-f)$ to units in $R$, with aid of bi-orthogonality. Moreover we shall see that regularity appears quite naturally in this setting.

Recall that if $(1-e) x(1-f) R=(1-e) R$ and $R(1-e) x(1-f)=R(1-f)$ then we can find $y, z \in R$ such that $(1-e) x(1-f) y=(1-e)$ and $z(1-e) x(1-f)=(1-f)$. This shows that

$$
[(1-e) x(1-f)][(1-f)) y(1-e)]=(1-e) \text { and }[(1-f) z(1-e)][(1-e) x(1-f)]=(1-f) .
$$

The associative law now ensures that $(1-f) y(1-e)=(1-f) z(1-e)$ and $(1-e) x(1-f)$ is a "semi-invertible" in the skew corner ring $(1-e) R(1-f)$.

Let us now use the identities of (6) in our search for bi-orthogonality. Indeed if we take $\alpha=$ $(1-e) x(1-f)$ and $\beta=(1-f) y(1-e)$ then we know that $\alpha \beta=1-e$ and $\beta \alpha=1-f$ and we must select

$$
a b=e \text { and } b a=f .
$$

In order to satisfy the orthogonality conditions (4), it suffices to assume

$$
(1-f) b=0=b(1-e) \text { and } a(1-f)=0=(1-e) a .
$$

This says that $b=f b=b e$ and $a=a f=e a$. We now can easily check that

$$
\begin{aligned}
{[a+(1-e) x(1-f)][b+(1-f) y(1-e)] } & =a b+0+0+[(1-e) x(1-f)][(1-f) y(1-e)] \\
& =e+(1-e)=1
\end{aligned}
$$

Likewise

$$
\begin{aligned}
{[b+(1-f) y(1-e)][a+(1-e) x(1-f)] } & =b a+[(1-f) y(1-e)][(1-e) x(1-f)] \\
& =f+(1-f)=1 .
\end{aligned}
$$


Because $a b=e$ we further get $a b a=e a=a$ and $b a b=b e=b$. In other words, $a$ and $b$ are regular and $b=a^{+}$is a reflexive inverse of $a$.

We can satisfy the stronger orthogonality conditions (8) by choosing $e=a a^{-}$and $f=a^{=} a$, where $a^{=}$is a 1-inverse of $a$. With this choice, we have

$$
b=b e=f b e=a^{=} a b a a^{-}=a^{=} a a^{-},
$$

which clearly is a reflexive inverse of $a$.

The two units now take the form

$$
\begin{aligned}
& {\left[a+\left(1-a a^{-}\right) x\left(1-a^{=} a\right)\right]\left[a^{=} a a^{-}+\left(1-a^{+} a\right) y\left(1-a a^{-}\right)\right]=1} \\
& =\left[a^{=} a a^{-}+\left(1-a^{+} a\right) y\left(1-a a^{-}\right)\right]\left[a+\left(1-a a^{-}\right) x\left(1-a^{=} a\right)\right]
\end{aligned}
$$

If we replace $a^{-}$and $a^{=}$both by $a^{+}$, then

$$
\left[a+\left(1-a a^{+}\right) x\left(1-a^{+} a\right)\right]^{-1}=a^{+}+\left(1-a^{+} a\right) y\left(1-a a^{+}\right)
$$

provided $\left(1-a a^{+}\right) x\left(1-a^{+} a\right) y=\left(1-a a^{+}\right)$and $R\left(1-a a^{+}\right) x\left(1-a^{+} a\right)=R\left(1-a^{+} a\right)$.

The analogue for two regular elements $a$ and $b$ is given by [6].

Lemma 2.2. If $a R=b R$ and $R a=R b$, then there exist units $u$ and $v$ such that $a u=b=v a$.

Proof. Indeed, $u=a^{+} b+\left(1-a^{+} a\right)\left(1-b^{+} b\right)$ and $v=a b^{+}+\left(1-a a^{+}\right)\left(1-b b^{+}\right)$with inverses $u^{-1}=$ $b^{+} a+\left(1-b^{+} b\right)\left(1-a^{+} a\right)$ and $v^{-1}=b a^{+}+\left(1-b b^{+}\right)\left(1-a a^{+}\right)$.

We now turn to a special case where another idempotent $f$ will be introduced.

\section{Relation between types of invertibility}

The question of invertibility of a sum is closely related to that of "generalized invertibility", such as regularity or the existence of a group inverse. Our aim is to express one type of invertibility for one element in terms of a "lower level" of invertibility for another element [8].

We shall focus on the regularity and strongly-pi-regularity of the elements $u=a g+1-a a^{-}$and $v=a+\left(1-a a^{-}\right) h$, when $a$ is regular, and in particular on the (one-sided) invertibility of these elements. These are not equivalent unless $g$ or $h$ is a unit. We shall see that the former is much simpler to handle than the latter. Needless to say, the case where $g=1$ is of special interest, and can be used to characterize the invertibility of two by two block matrices.

We begin by observing that if $p$ is a unit and $m=p a q$, then $p^{-1} m p=a(q p)$ and as such that $p^{-1} m^{d} p=[a(q p)]^{d}$. In other words the search for $m^{d}$ reduces to that of finding $(a g)^{d}$ where $g=q p$. A parallel result holds if $q$ is a unit.

We begin with an easier case. 


\subsection{The element $a g+1-a a^{-}$}

To investigate the regularity or invertibility of a sum $a+b$, where $a$ is regular, there are two approaches we can take, namely

(i) a direct approach;

(ii) use the Pierce's Decomposition of Theorem 2.2 of [7].

The latter, however, requires that $\mu=1+a^{+} b$ and $\nu=1+b a^{+}$both be units, which may not be satisfied in all cases. We first take the direct approach.

\section{Direct approach}

Suppose that $\left(a g+1-a a^{-}\right) x\left(a g+1-a a^{-}\right)=a g+1-a a^{-}$. Premultiplication by $a a^{-}$followed by post multiplication by a gives agxaga =aga. Next, premultiplication by $1-a a^{-}$followed by post multiplication by $a$ gives $\left(1-a a^{-}\right) x a g a=0$ and thus $x a g a=a a^{-} x a g a$. Substituting this into the previous identity we arrive at

$$
(a g a)\left(a^{-} x\right)(a g a)=a g a
$$

and hence aga must be regular.

Conversely suppose that $(a g a) z(a g a)=a g a$. Then it can be verified that $\left(a g+1-a a^{-}\right)^{-}=$ $a z a a^{-}+1-a z a g$, which, needless to say, is not obvious.

Let us now use the second option.

\section{Pierce's decomposition}

Consider $u=\left(1-a a^{-}\right)+a g=A+B$. Then $A^{+} B=\left(1-a a^{-}\right) a g=0$ and $\mu=1+A^{+} B=1$. We then have $y=\left(1-A A^{+}\right) B \mu^{-1}\left(1-A^{+} A\right)=a a^{-} a g a a^{-}=a g a a^{-}$. Because $a\{a g a\}^{-} \subseteq\left\{a g a a^{-}\right\}^{-}$and $a^{-}\left\{a g a a^{-}\right\}^{-} \subseteq\{a g a\}^{-}$we may state [7, Theorem 2.2],

Lemma 3.1. The following are equivalent:

(a) $a g+1-a a^{-}$is regular.

(b) $\mathrm{agaa}^{-}$is regular.

(c) aga is regular.

In which case

$$
\left(a g+1-a a^{-}\right)^{-}=a(a g a)^{-} a a^{-}+1-a(a g a)^{-} a g .
$$

Let us now turn to the left and right indices of the element $u=a g+1-a a^{-}$, and extend the work of $[3]$.

Theorem 3.1. Let $u=a g+1-a a^{-}$.

(a) $\operatorname{rind}(u) \leq k$ iff $(a g)^{k+1} a R=(a g)^{k} a R$.

(b) $\operatorname{lind}(u) \leq k$ iff $R(a g)^{k+1} a=R(a g)^{k} a$. 
(c) $\operatorname{ind}(u) \leq k$ iff $(a g)^{k+1} a R=(a g)^{k} a R$ and $R(a g)^{k+1} a=R(a g)^{k} a$, in which case $u$ is strongly-piregular, and the Drazin inverse $u^{d}$ exists and is given by

$$
u^{d}=s u^{k} r^{k}=s^{k} u^{k} r=u^{k} r^{k+1}=s^{k+1} u^{k}
$$

where $u^{k+1} r=u^{k}=s u^{k+1}$.

Proof. (a). First we observe [3] that

$$
\left.u^{k}=(a g)^{k}+\left[(a g)^{k-1}+\cdots+a g+1\right]\left(1-a a^{-}\right)\right] .
$$

and hence that

$$
u^{k} a=(a g)^{k} a \text { and }\left(1-a a^{-}\right) u^{k}=1-a a^{-} .
$$

Now suppose that $u^{k+1} r=u^{k}$. Then $u^{k+1} r a=u^{k} a=(a g)^{k} a$. Premultiplication by $1-a a^{-}$gives $\left(1-a a^{-}\right) u^{k+1} r a=0$ and thus

$$
\left(1-a a^{-}\right) r a=0 .
$$

Substituting this in $u^{k+1} r a=u^{k} a$ gives $(a g)^{k+1} r a=(a g)^{k} a$. On account of (11) this shows that $(a g)^{k+1} a\left(a^{-} r a\right)=(a g)^{k} a$ and $(a g)^{k+1} a R=(a g)^{k} a R$.

For the converse suppose that $(a g)^{k+1} a y=(a g)^{k} a$. It can now be verified directly that

$$
u^{k+1}\left[a y a^{-}+1-a y g\right]=u^{k},
$$

as desired.

(b) Suppose $s u^{k+1}=u^{k}$. Postmultiplication by $a$ gives $s(a g)^{k+1} a=s u^{k+1} a=u^{k} a=(a g)^{k} a$, ensuring that $R(a g)^{k+1} a=R(a g)^{k} a$.

Conversely let $z(a g)^{k+1} a=(a g)^{k} a$. We will show that $\left[a a^{-} z a a^{-}+1-a a^{-} z a g\right] u^{k+1}=u^{k}$. Indeed,

$$
\begin{aligned}
\left(a a^{-} z a a^{-}+1-a a^{-} z a g\right) u^{k+1}= & a a^{-} z(a g)^{k+1}+\sum_{1}^{k} a a^{-} z(a g)^{j}\left(1-a a^{-}\right)+ \\
& +u^{k+1}-a a^{-} z(a g)^{k+2}-a a^{-} z a a^{-} \sum_{1}^{k+1}(a g)^{j}\left(1-a a^{-}\right) \\
= & a a^{-} z(a g)^{k+1}-a a^{-} z a a^{-}(a g)^{k+1}\left(1-a a^{-}\right)+\sum_{0}^{k}(a g)^{j}\left(1-a a^{-}\right) \\
= & a a^{-} z(a g)^{k+1} a a^{-}+(a g)^{k}\left(1-a a^{-}\right)+\sum_{0}^{k-1}(a g)^{j}\left(1-a a^{-}\right) \\
= & a a^{-} z(a g)^{k+1} a a^{-}-(a g)^{k} a a^{-}+u^{k}=u^{k}
\end{aligned}
$$

(c) Clear from (a), (b) and [8].

It should be noted that a knowledge of $r$ and $s$ such that $u^{k}=u^{k+1} r=s u^{k+1}$ will give us the elements $y$ and $z$ such that $z(a g)^{k+1} a=(a g)^{k} a=(a g)^{k+1} a y$, and conversely. Indeed

(I) $r=a y a^{-}+1-a y g$ and $s=a a^{-} z a a^{-}+1-a a^{-} z a g$

(II) $y=a^{-} r a$ and $z=s$. 
When $g$ is a unit we can simplify the above conditions somewhat. In fact, $(a g)^{k} a R=(a g)^{k} a g R=$ $(a g)^{k+1} R$ and hence

$$
\exists r: u^{k+1} r=u^{k} \Leftrightarrow \exists \lambda:(a g)^{k+2} \lambda=(a g)^{k+1} .
$$

In this case $(a g)^{k+1} a\left(a^{-} r a\right)=(a g)^{k} a$ becomes $(a g)^{k+1} a g\left(g^{-1} a^{-} r a g\right)=(a g)^{k} a g$ or $(a g)^{k+2} \lambda=(a g)^{k+1}$, where $\lambda=g^{-1}\left(a^{-} r a\right) g$.

Similarly $R(a g)^{k+1} a=R(a g)^{k} a$ if and only if $R(a g)^{k+2}=R(a g)^{k+1}$, and

$$
\exists s: s u^{k+1}=u^{k} \Leftrightarrow \exists \mu: \mu(a g)^{k+2}=(a g)^{k+1} .
$$

Indeed, $s(a g)^{k+1} a=(a g)^{k} a$ is equivalent to $\mu(a g)^{k+2}=(a g)^{k+1}$ where $\mu=s$.

If both hold, $u^{d}=s u^{k} r^{k}=s^{k} u^{k} r$ and

$$
(a g)^{d}=(\mu)^{k+1}(a g)^{k+1} \lambda=s^{k+1}(a g)^{k+1}\left(g^{-1} a^{-} \text {rag }\right) .
$$

Let us examine the special cases where the index equals $k=0, k=1$ or when $g=1$.

Corollary 3.1. (a) $a g+1-a a^{-}$has a right inverse iff $a g a R=a R$.

(b) $a g+1-a a^{-}$has a left inverse iff Raga $=R a$.

(c) $a g+1-a a^{-}$is a unit iff $a g a R=a R$ and $R a g a=R a$, in which case

$$
u^{-1}=a y a^{-}+1-a y g=a a^{-} z a a^{-}+1-a a^{-} z a g,
$$

where agay $=a=z a g a$.

(d) $a g+1-a a^{-}$has a group inverse iff $(a g)^{2} a R=a g a R$ and $R(a g)^{2} a=R a g a$ in which case

$$
u^{\#}=a a^{-} z a g a y a^{-}+a a^{-} z a g a a^{-}+a g+1-a a^{-} z a g a y g-a a^{-} z a g a g-a a^{-},
$$

where $(a g)^{2} a y=a g a=z(a g)^{2} a$.

Proof. (d). Since $r u^{2}=u=u^{2} s$ then $u$ exists and $u^{\#}=r u s$. The formula is obtained by performing the desired calculations.

When $g$ is a unit we know that $(a g)^{k} a R=(a g)^{k+1} R$, and $R(a g)^{k} a=R(a g)^{k+1}$. This gives

Corollary 3.2. If $g$ is a unit,

(a) $a g+1-a a^{-}$has a right inverse iff $(a g)^{2} R=a R$.

(b) $a g+1-a a^{-}$has a left inverse iff $R(a g)^{2}=R a g$.

(c) $a g+1-a a^{-}$is a unit iff $(a g)^{\#}$ exists, in which case

$$
u^{-1}=a g \lambda a^{-}+1-a g \lambda g=a a^{-} \mu a a^{-}+1-a a^{-} \mu a g,
$$

where $(a g)^{2} \lambda=a=\mu a g a$.

Moreover,

$$
(a g)^{\#}=u^{-1} a a^{-} u^{-1} a g .
$$


Proof. These follow from the previous results. It remains to show the expression for $(a g)^{\#}$. Since $a g=z(a g)^{2}=(a g)^{2}\left(g^{-1} y g\right)$ then $(a g)^{\#}=z(a g)\left(g^{-1} y g\right)$ (see [5]). From $y=a^{-} r a$ and $z=s$, with $u r=1=s u$, we obtain $(a g)^{\#}=s a g g^{-1} a^{-} r a g=s a a^{-}$rag. Because $r=s=u^{-1}$ this takes the form $(a g)^{\#}=u^{-1} a a^{-} u^{-1} a g$.

When $g=1$, Lemma 3.1, Corollary 3.1 and Corollary 3.2, collapse to

Corollary 3.3. $\quad$ 1. $a+1-a a^{-}$is regular iff $a^{2} a^{-}$is regular iff $a^{2}$ is regular.

2. $a+1-a a^{-}$has a right inverse iff $a^{2} R=a R$.

3. $a+1-a a^{-}$has a left inverse iff $R a^{2}=R a$.

4. The following are equivalent:

(a) $w=a^{2} a^{-}+1-a a^{-}$is a unit;

(b) $k=a^{-} a^{2}+1-a^{-} a$ is a unit;

(c) $u=a+1-a a^{-}$is a unit;

(d) $v=a+1-a^{-} a$ is a unit;

(e) $a$ has a group inverse, in which case

$$
u^{-1}=a^{\#} a a^{-}+1-a a^{\#}
$$

and

$$
a^{\#}=w^{-1} a k^{-1}=u^{-2} a=a v^{-2}=u^{-1} a v^{-1} .
$$

5. $a+1-a a^{-}$has a group inverse iff $a^{3} R=a^{2} R$ and $R a^{3}=R a^{2}$, in which case

$$
u^{\#}=a^{d} a a^{-}+a^{2} a^{d} a^{-}+a+1-a^{d} a-a^{2} a^{d}-a a^{-}
$$

and

$$
a^{d}=u^{\#} a^{2} a^{-} u^{\#} a .
$$

Proof. (4). The equivalences follow from [9, 11]. In order to prove the expressions given in (17), write $u=1-a a^{-}\left(a a^{-}-a\right)=1-x y$, with $x=a a^{-}$and $y=a a^{-}-a$ and assume it is a unit. Then $w^{-1}=(1-y x)^{-1}=1+y u^{-1} x=1+\left(a a^{-}-a\right) u^{-1} a a^{-}$and $w^{-1} a=u u^{-1} a a^{-} u^{-1} a-a u^{-1} a=$ $\left(u+a a^{-}-a\right) u^{-1} a=u^{-1} a$. Similarly, $a k^{-1}=a v^{-1}$. Since $u a=a v$ then $a v^{-1}=u^{-1} a$, and $a^{\#}=$ $w^{-1} a k^{-1}=u^{-1} a v^{-1}=u^{-2} a$.

(5). If in addition $a^{3} \lambda=a^{2}=\mu a^{3}$ then $a^{d}=(\mu)^{2} a^{2} \lambda=s^{2} a^{2} a^{-} r a$. Selecting $r=s=u^{\#}$ then gives

$$
a^{d}=u^{\#} a^{2} a^{-} u^{\#} a .
$$

The expression for $u^{\#}$ given in (18) follows directly from (16). 


\section{Remarks}

1. If $n$ is regular nilpotent of index $r$, then the power $r$ of $u=n+1-n n^{-}$is regular. Indeed, since $u^{r}=\left(n^{r-1}+\ldots n+1\right)\left(1-n n^{-}\right)=(1-n)^{-1}\left(1-n n^{-}\right)$then $(1-n) u^{r}=1-n n^{-}$from which $u^{r}(1-n) u^{r}=u^{r}$. We note that $u$ may not be regular. As an example, take $N=\left[\begin{array}{ll}0 & 1 \\ 0 & 0\end{array}\right]$, $N^{-}=\left[\begin{array}{ll}0 & 0 \\ 1 & 0\end{array}\right]$ in the ring of $2 \times 2$ matrices over $\mathbb{Z}_{4}$. The matrix $U=N+I_{2}-N N^{-}=\left[\begin{array}{ll}2 & 1 \\ 0 & 1\end{array}\right]$ is not regular since $\operatorname{det}(U)=2$ is not regular in $\mathbb{Z}_{4}$.

2. It is of interest to see when $n g+\left(1-n n^{-}\right)$will be regular, when $n$ is regular nilpotent and $g$ is arbitrary.

3. We can also directly derive the expression (18). Set $w=a^{2} a^{-}+1-a a^{-}$. Since $i(a) \leq 2$ then $i(w) \leq 1$, and $w^{\#}=a^{d} a a^{-}+1-a a^{-}$, using [8, Theorem 3.5]. We may write $w=1-\alpha \beta$, with $\alpha=a a^{-}-a$ and $\beta=a a^{-}$and note that $u=1-\beta \alpha$. Using [2, Theorem 3.5], the indices of $u$ and $w$ are equal, and $u^{\#}=(1-\beta \alpha)^{\#}=1+a a^{-}\left[w^{\#}-\left(1-w w^{\#}\right)\right]\left(a a^{-}-a\right)$. The expression follows.

\subsection{The other twin $v=a+\left(1-a a^{-}\right) h$}

Let us now turn to the twin element $v=a+\left(1-a a^{-}\right) h=a+e h$. The computations will be much harder because of the lack of simple orthogonality and the absence of telescoping. We again begin with the question of regularity.

\section{Direct approach}

Suppose $a$ is regular and that $e=1-a a^{-}, f=1-a^{-} a$. Clearly $e a=0=a f$.

We wish to find out when $a+\left(1-a a^{-}\right) h$ is also regular and begin with the necessary conditions. First, let

$$
(a+e h) x(a+e h)=a+e h .
$$

Pre and post multiplication by $e$ and $f$ respectively gives

$$
\text { ehxehf }=e h f \text {. }
$$

On the other hand, pre-multiplication by $a^{-} a$ and post-multiplication by $f$ gives

$$
(1-f) x e h f=0 \text { or } \quad x e h f=f x e h f .
$$

Plugging back into (21) gives

$$
(e h f) x(e h f)=e h f \text {. }
$$

and ehf must be regular.

Suppose now that $a a^{=} a=a$ and that $e^{\prime}=1-a a^{=}, f^{\prime}=1-a^{=} a$. We may state

Lemma 3.2. For any $h$, ehf is regular iff $e^{\prime} h f^{\prime}$ is regular. 
Proof. Observe that $e^{\prime} e=e^{\prime}, e e^{\prime}=e$ and $f f^{\prime}=f^{\prime}, f^{\prime} f=f$. Now if $(e h f) x(e h f)=e h f$, then $e^{\prime}(e h f) x(e h f) f^{\prime}=e^{\prime}(e h f) f^{\prime}$ or $e^{\prime} h f^{\prime}(f x e) e^{\prime} h f^{\prime}=e^{\prime} e h f f^{\prime}=e^{\prime} h f^{\prime}$, and hence $f x e$ is an inner inverse of $e^{\prime} h f^{\prime}$.

For the sufficient condition we need the form of the desired inner inverse in terms of $(e h f)^{-}$. To see what possible contenders are feasible, we shall first turn to the alternative approach, which uses Pierce's Decomposition [7].

\section{Pierce's Decomposition}

Let $e^{\prime}=1-a a^{+}, f^{\prime}=1-a^{+} a$ and consider $u=a+e^{\prime} h=A+B$. Then $A^{+} B=0$. We may apply $[7$, Corollary 2.2] with $y=\left(1-A A^{+}\right) B^{-1}\left(1-A^{+} A\right)=e^{\prime} h f^{\prime}$ which implies that

$$
a+e^{\prime} h \text { is regular iff } y=e^{\prime} h f^{\prime} \text { is regular, }
$$

in which case we have the reflexive inverse

$$
\left(a+e^{\prime} h\right)^{+}=\left[a^{+}+f^{\prime}\left(e^{\prime} h f^{\prime}\right)^{+} e^{\prime}\right]\left(1-e^{\prime} h a^{+}\right)=a^{+}+f^{\prime}\left(e^{\prime} h f^{\prime}\right)^{+} e^{\prime}\left(1-h a^{+}\right) .
$$

Based on the form of this inner inverse we can verify that when ehf is regular, with $e=1-a a^{-}, f=$ $1-a^{-} a$, then

$$
(a+e h)^{-}=a^{-}+f(e h f)^{-} e\left(1-h a^{-}\right) .
$$

On account of Lemma 3.2 we may now state

Lemma 3.3. The following are equivalent:

1. $a+\left(1-a a^{-}\right) h$ is regular.

2. $\left(1-a a^{-}\right) h\left(1-a^{-} a\right)$ is regular.

3. $\left(1-a a^{+}\right) h\left(1-a^{+} a\right)$ is regular.

4. $a+\left(1-a a^{+}\right) h$ is regular.

When $h$ and $g=h^{-1}$ are units, and we set $u=a g+1-a a^{-}$then $v=u h=u g^{-1}$. Hence $v$ is regular iff $u$ is regular and $v^{+}=g u^{+}, v v^{+}=u u^{+}$and $v^{+} v=g u^{+} u h$.

An associated element is $w=a g a a^{-}+1-a a^{-}=1-(1-a g) a a^{-}$. From (1), we know that $w$ is regular iff $u=1-a a^{-}(1-a g)$ is regular and that $w^{-}=1+(1-a g) u^{-} a a^{-}$.

For later use we present the case where $g=1=h$ and $u=v=a+1-a a^{-}$. We obtain

Corollary 3.4. $\quad$ 1. $u^{-}=a\left(a^{2}\right)^{-} a a^{-}+1-a\left(a^{2}\right)^{-} a$

2. $u^{-}=a^{-}+\left(1-a a^{-}\right)\left[\left(1-a a^{-}\right)\left(1-a^{-} a\right)\right]^{-}\left(1-a a^{-}\right)\left(1-a^{-}\right)$

3. $u u^{-}=1-a a^{-}+a+a^{2}\left(a^{2}\right)^{-} a\left(a^{-}-1\right)$.

4. $u^{-} u=1-a a^{-}+a+a\left(a^{2}\right)^{-} a^{2}\left(a^{-}-1\right)$. 
To obtain a reflexive inverse of $u=a+1-a a^{-}$, we can either use $u^{+}=u^{-} u u^{-}$, or use Jacobson's lemma [2]. Set $u=1-B A$, where $B=a a^{-}$and $A=1-a$. Also let $z=1-A B=a^{2} a^{-}+1-a a^{-}$, which contains a bi-orthogonal splitting. We now recall $[2]$

$$
u^{+}=(1-B A)^{+}=1+B\left[z^{+}-\left(1-z^{+} z\right)\left(1-z z^{+}\right] A .\right.
$$

Now, bi-orthogonality easily yields a reflexive inverse $z^{+}=a a^{-}\left(a^{2} a^{-}\right)^{+} a a^{-}+1-a a^{-}$, which may be substituted to give a second reflexive inverse of $u$.

\section{Remarks}

1. It is not known if a more compact reflexive inverse of $a+1-a a^{-}$can be found.

2. Writing $u=a+e$ with $e=1-a a^{+}$, we see that $u=\nu(a+e f)$, where $\nu=1+e a^{+}$and $f=1-a^{+} a$. On the other hand, writing $u=e+a$, we see that $u=\nu^{\prime}\left(e+a^{2} a^{+}\right)$, where $\nu^{\prime}=1+a e$. Hence

$$
\nu(a+e f)=\nu^{\prime}\left(e+a^{2} a^{+}\right)
$$

and therefore a reflexive inverse of $a+e f$ can be obtained via $\left(a^{2} a^{+}+e\right)^{+}=a a^{+}\left(a^{2} a^{+}\right)^{+} a a^{+}+e$. We may, as such, relate $(a+e)^{+}$to $\left(a^{2} a^{+}\right)^{+}$, but the relation is not very "pretty".

3. It would be of interest to show directly that $a h^{-1} a$ is regular iff $\left(1-a a^{-}\right) h\left(1-a^{-} a\right)$ is regular.

4. It is not obvious why $\left(1-a a^{-}+a\right)+a^{2}\left(a^{2}\right)^{+}(1-a)$ is idempotent.

5. It is not clear if any simplification occurs when $h a=a h$ in $v=a+\left(1-a a^{-}\right) h$.

We next turn to one-sided inverses.

\subsection{One-sided inverses}

Theorem 3.2. Let $a$ be regular and let $e=1-a a^{-}$for some $a^{-}$. Then

1. $a+e h$ has a right inverse iff there exists an inner inverse $a^{=}$and an associated idempotent $f=1-a^{=} a$ such that

$$
e h(1-f)=0 \quad \text { and } \quad \text { ehf } R=e R .
$$

2. $a+e h$ is a unit if in addition

$$
R f=\operatorname{Reh} f
$$

and conversely.

Proof. (1). Let $[a+e h] x=1$. Pre-multiplying this by $1-e$ shows that $a x=1-e$ and thus $a x a=a$, so that $x$ is an inner inverse of $a$, and we set $1-f=x a$. Next we post-multiply by $a$, giving $a+e h x a=a$. Consequently, $e h(1-f)=0$ and $e h f=e h$.

We then have $R=[a+e h] R \subseteq a R \dot{+} e h R=a R \dot{+} e h f R \subseteq a R \dot{+} e R=R$. Since ehf $R \subseteq e R$ and the sums are direct we may conclude that ehf $R=e R$.

Recall that if $R=a R \dot{+} b R=a R \dot{+} c R$, then $b R \subseteq c R \Rightarrow b R=c R$. 
We moreover have that $\operatorname{ehf} R=e h R$ and may also conclude that $e h R=e R$ exactly when $(1-$ e) $R+h R=R$, i.e. $a R+h R=R$.

Conversely, let ehfy $=e$ and suppose $e h=e h f$. Then $(a+e h)\left((1-f) a^{-}+f y\right)=a(1-f) a^{-} a f y+$ $e h(1-f) a^{-}+e h f y=a a^{-}+0+e h a^{-}-e h f a^{-}+e=1$. As such $a+e h$ has a right inverse. Note that $(1-f) a^{-}=a^{=} a a^{-}$, which is a reflexive inverse of $a$. Units via reflexive inverses have been studied in $[6]$.

(2). Suppose now that also $x[a+e h]=1$. That is, $x a+x e h=1$, which gives $x e h f=f$ and therefore $R e h f=R f$.

Conversely, suppose that in addition to $e h f=e h$ we also have $f=y e h f$. Then $\left[a^{=}(1-e)+y e\right](a+$ $e h)=a^{=} a+y e h=1$.

We note that $b=a^{=} a a^{-}, \alpha=e h=e h f$ and $\beta=f y=$ fye.

\section{Plus-Orthogonality}

An important method of investigating regularity is via +-orthogonality [7], [10].

Lemma 4.1. (a) If $p$ is regular and $p^{+} q=0=q p^{+}$then $p+q$ regular implies that $q$ is regular. Indeed, $(p+q)^{-}=q^{-}$.

(b) If $q$ is regular and in addition $q^{+} p=0=p q^{+}$then $p+q$ is regular and $(p+q)^{+}=p^{+}+q^{+}$.

We now note that the Brown-McCoy shift method $[1,4]$ is a special application of +-orthogonality. Indeed, let $m=t+n$, where $t=m \hat{m} m$ and $n=\beta(m)=m-t$. Then

1. If $n$ is regular, then $f n^{-} e=n^{=}$is a 1-inverse of $n$.

2. et $=0=t f$.

3. If $t t^{-} t=t$ ( $t$ is regular) then $t^{=}=(1-f) t^{-}(1-e)$ is an inner inverse of $t$.

4. $t^{=} n=0=n t^{=}$and $t^{=} e n=0=n f t^{=}$.

5. $n^{=} t=\left(f n^{=} e\right) t=0=t\left(f n^{-} e\right)=t n^{=}$.

6. If $m$ is regular so is $n$, because $n m^{-} n=e m m^{-} m f=e m f=n$.

7. If $n$ is regular then $n^{=}$exists and $m$ is regular with inner inverse $m^{=}=t^{=}+n^{=}$.

In fact $m m^{=}=(t+n)\left(t^{=}+n^{+}\right)=t t^{=}+n n^{+}$and $\left(t t^{=}+n n^{=}\right)(t+n)=t+n$.

8. If $t^{+}$is a reflexive inverse of $t$ then so is $t^{=}=(1-f) t^{+}(1-e)$

9. $\hat{m}+x$ is an outer inverse of $m$ iff

$$
x-x m x=(1-f) x+x(1-e) .
$$

This shows that the shift procedure is really a special application of +-orthogonality. As a special application of +-orthogonality we examine the matrix case. 


\section{The matrix case}

Consider $M=\left[\begin{array}{ll}a & c \\ b & d\end{array}\right]$, where we assume that one of the blocks is regular. For the sake of symmetry it is best to assume that $d$ or $a$ is regular. Assuming the former, we may now use equivalence to "improve" the off diagonal entries, making them closer to "being orthogonal". We shall apply block splittings to matrices as follows.

Suppose that $d^{+} c=0=b d^{+}$and split $M$ as

$$
M=\left[\begin{array}{ll}
0 & 0 \\
0 & d
\end{array}\right]+\left[\begin{array}{ll}
a & c \\
b & 0
\end{array}\right]=P+Q
$$

with $P^{+} Q=0=Q P^{+}$. Hence, by Lemma 4.1, if $M$ is regular so is $Q=\left[\begin{array}{cc}a & c \\ b & 0\end{array}\right]$.

Now suppose that $c$ and $b$ are regular and that $c^{+} a=0=a b^{+}$. Then we can further split

$$
Q=\left[\begin{array}{ll}
0 & c \\
b & 0
\end{array}\right]+\left[\begin{array}{ll}
a & 0 \\
0 & 0
\end{array}\right]=R+S
$$

with $R^{+} S=0=S R^{+}$. Hence if $Q$ is regular so is $S$, i.e., $a$ must be regular.

For the reverse, suppose that $a$ is regular and that $a^{+} c=0=b a^{+}$. Then $S^{+} R=0=R S^{+}$with $S^{+}=\left[\begin{array}{cc}a^{+} & 0 \\ 0 & 0\end{array}\right]$. Hence if both conditions $c^{+} a=0=a b^{+}$and $a^{+} c=0=b a^{+}$hold then $Q=R+S$ is regular with $Q^{+}=(R+S)^{+}=R^{+}+S^{+}=\left[\begin{array}{cc}a^{+} & b^{+} \\ c^{+} & 0\end{array}\right]$.

We can go another step backwards if we the assume that $Q^{+} P=0=P Q^{+}$. This means that $M$ is regular with

$$
M^{+}=P^{+}+Q^{+}=\left[\begin{array}{ll}
a^{+} & b^{+} \\
c^{+} & d^{+}
\end{array}\right]
$$

Using the factorization

$$
\left[\begin{array}{cc}
1 & -c d^{+} \\
0 & 1
\end{array}\right] M\left[\begin{array}{cc}
1 & 0 \\
-d^{+} b & 1
\end{array}\right]=\left[\begin{array}{cc}
\zeta & c f \\
e b & d
\end{array}\right]=A
$$

where $e=1-d d^{+}, f=1-d^{+} d$ and $\zeta=a-c d^{+} b$ is the Schur complement of $d$ in $M$, it is clear that $M$ is regular iff $A$ is regular.

Consider, therefore, the matrix $A=\left[\begin{array}{cc}\zeta & c f \\ e b & d\end{array}\right]$, and observe that $d^{+}(e b)=0=c f d^{+}$. We may split

$$
A=\left[\begin{array}{cc}
\zeta & c f \\
e b & d
\end{array}\right]=\left[\begin{array}{ll}
0 & 0 \\
0 & d
\end{array}\right]+\left[\begin{array}{cc}
\zeta & c f \\
e b & 0
\end{array}\right]=B+C .
$$

Then $B^{+} C=0=C B^{+}$and hence if $A$ and $B$ are regular so is $C$.

To repeat, we must assume that $e b$ and $c f$ are regular. However the obvious splitting

$$
C=\left[\begin{array}{cc}
\zeta & c f \\
e b & 0
\end{array}\right]=\left[\begin{array}{cc}
0 & c f \\
e b & 0
\end{array}\right]+\left[\begin{array}{cc}
\zeta & 0 \\
0 & 0
\end{array}\right]=K+L
$$


is not usefull since $(c f)^{+} \zeta$ and $\zeta(e b)^{+}$need not vanish. Instead we reduce $C$ further. We first define the idempotents:

$$
\begin{array}{ll}
g=1-(e b)(e b)^{+} & h=1-(e b)^{+}(e b) \\
p=1-(c f)(c f)^{+} & q=1-(c f)^{+}(c f) .
\end{array}
$$

We now have

$$
\left[\begin{array}{cc}
1 & -p \zeta(e b)^{+} e \\
0 & 1
\end{array}\right]\left[\begin{array}{cc}
\zeta & c f \\
e b & d
\end{array}\right]\left[\begin{array}{cc}
1 & 0 \\
-f(c f)^{+} \zeta & 1
\end{array}\right]=\left[\begin{array}{cc}
w & c f \\
e b & d
\end{array}\right]=X
$$

and can now proceed to split

$$
X=\left[\begin{array}{cc}
0 & c f \\
e b & d
\end{array}\right]+\left[\begin{array}{cc}
w & 0 \\
0 & 0
\end{array}\right]
$$

where $w=p \zeta h$.

Now it is clear that

$M$ is regular iff $A$ is regular iff $X$ is regular.

We now claim that $X$ is regular iff $w$ is regular. To do this we repeat the above splitting with $\zeta$ replaced by $w$, i.e.,

$$
X=\left[\begin{array}{cc}
w & c f \\
e b & d
\end{array}\right]=\left[\begin{array}{ll}
0 & 0 \\
0 & d
\end{array}\right]+\left[\begin{array}{cc}
w & c f \\
e b & 0
\end{array}\right]=Y+Z
$$

Hence if $X$ and $Y$ are regular then so is $\left[\begin{array}{cc}w & c f \\ e b & 0\end{array}\right]$. We now however, can proceed with a second splitting

$$
Z=\left[\begin{array}{cc}
w & c f \\
e b & 0
\end{array}\right]=\left[\begin{array}{cc}
0 & c f \\
e b & 0
\end{array}\right]+\left[\begin{array}{cc}
w & 0 \\
0 & 0
\end{array}\right]=U+V
$$

Because $w=p \zeta h$, we see that $(c f)^{+} w=0=w(e b)^{+}$and $U^{+} V=0=V U^{+}$so that we have a +-orthogonal splitting.

Lastly, since $Z$ and $U$ are regular, so is $V$ and thus $w$ is regular.

For the converse we need that $V^{+} U=0=U V^{+}$.

Assume $w$ is regular. Because of the form of $w$ we know that $h w^{+} p$ is a reflexive inverse of $w$, so we can take $V^{+}=\left[\begin{array}{cc}h w^{+} p & 0 \\ 0 & 0\end{array}\right]$. Since $h w^{+} p(c f)=0=(e b) h w^{+} p$ we see that $V^{+} U=0=U V^{+}$and we may take

$$
Z^{+}=U^{+}+V^{+}=\left[\begin{array}{cc}
h w^{+} p & (e b)^{+} e \\
f(c f)^{+} & 0+
\end{array}\right]
$$

entailing that

$$
X^{+}=Y^{+}+Z^{+}=\left[\begin{array}{cc}
h w^{+} p & (e b)^{+} e \\
f(c f)^{+} & d^{+}
\end{array}\right] \text {. }
$$

The above splitting also provides a way to compute a 1-inverse of a matrix.

As an example, consider the matrix $M=\left[\begin{array}{cc}29 & 2 \\ 3 & 10\end{array}\right]=\left[\begin{array}{cc}a & c \\ b & d\end{array}\right]$ over the commutative ring $\mathbb{Z}_{35}$. Since $\operatorname{gcd}\left(a^{2}, 35\right)$ always divides $a$, for all $a \in \mathbb{Z}_{35}$, as 35 is the product of two distinct primes, then 
$a^{2} x=a \bmod 35$ is always consistent. That is, $\mathbb{Z}_{35}$ is a regular ring, and so is the ring of $n \times n$ matrices over $\mathbb{Z}_{35}$.

We will use the above splitting and notation to construct a 1-inverse of $M$.

Firstly, we obtain, for $d^{+}=5$,

$$
e=21, f=21, \zeta=34 \text {. }
$$

Also $c f=7$ and $e b=28$, and we may take $(c f)^{+}=28$ and $(e b)^{+}=7$, giving

$$
p=15, h=15, w=20 .
$$

For $w^{+}=20$ we construct $V^{+}=\left[\begin{array}{cc}20 & 0 \\ 0 & 0\end{array}\right]$. For $U^{+}=\left[\begin{array}{cc}0 & (e b)^{+} \\ (c f)^{+} & 0\end{array}\right]$, we obtain $Z^{+}=\left[\begin{array}{ll}20 & 7 \\ 28 & 0\end{array}\right]$.

From this, we may take $X^{+}=\left[\begin{array}{cc}20 & 7 \\ 28 & 5\end{array}\right]$, and thefore

$$
A^{-}=\left[\begin{array}{cc}
1 & 0 \\
-f(c f)^{+} \zeta &
\end{array}\right] X^{+}\left[\begin{array}{cc}
1 & p \zeta(e b)^{+} e \\
0 & 1
\end{array}\right]=\left[\begin{array}{cc}
20 & 7 \\
28 & 26
\end{array}\right] \text {. }
$$

Finally,

$$
M^{-}=\left[\begin{array}{cc}
1 & 0 \\
-d^{+} b & 1
\end{array}\right] A^{-}\left[\begin{array}{cc}
1 & -c d^{+} \\
0 & 1
\end{array}\right]=\left[\begin{array}{cc}
20 & 17 \\
8 & 16
\end{array}\right]
$$

\section{Remarks}

We conclude with some remarks and open questions.

1. The regularity of the sum $a+\left(1-a a^{+}\right)$will be used in the study of block group inverses.

2. If $e$ and $f$ are idempotent, when is $e+f$ regular or a unit?

3. What other results on generalized inverses are there using skew corner rings?

4. Parallel results exist for $a+1-a^{-} a$.

\section{Acknowlegdment}

The authors gratefully acknowledge the suggestions and corrections of the referee.

\section{References}

[1] Brown, Bailey; McCoy, Neal H.; The maximal regular ideal of a ring. Proc. Amer. Math. Soc. 1, (1950), 165-171.

[2] Castro-González, N.; Mendes-Araújo, C.; Patrício, Pedro; Generalized inverses of a sum in rings. Bull. Aust. Math. Soc. 82 (2010), no. 1, 156-164.

[3] Hall, F. J.; Hartwig, R.E.; Algebraic properties of governing matrices used in Cesaro-Neumann iterations. Rev. Roumaine Math. Pures Appl. 26 (1981), no. 7, 959-978. 
[4] Hartwig, R.E.; Block generalized inverses. Arch. Rational Mech. Anal. 61 (1976), no. 3, 197-251.

[5] Hartwig, R.E.; More on the Souriau-Frame algorithm and the Drazin inverse. SIAM J. Appl. Math. 31 (1976), no. 1, 42-46.

[6] Hartwig, R.E.; Generalized inverses, EP elements and associates. Rev. Roumaine Math. Pures Appl. 23 (1978), no. 1, 57-60.

[7] Hartwig, R.E.; Patrício, P.; Puystjens, R.; Diagonalizing triangular matrices via orthogonal Pierce decompositions. Linear Algebra Appl. 401 (2005), 381-391.

[8] Patrício, P.; Hartwig, R.E.; The link between regularity and strong-pi-regularity. J. Aust. Math. Soc. 89 (2010), no. 1, 17-22.

[9] Patrício, P.; Hartwig, R.E.; The (2,2,0) group inverse problem. Appl. Math. Comput. 217 (2010), no. 2, 516-520.

[10] Patrício, P.; Puystjens, R.; About the von Neumann regularity of triangular block matrices. Linear Algebra Appl. 332/334 (2001), 485-502.

[11] Puystjens, R.; Hartwig, R.E.; The group inverse of a companion matrix. Linear and Multilinear Algebra 43 (1997), no. 1-3, 137-150. 